\title{
The effect of thermal radiation on singularities in the dark universe
}

\author{
I. Brevik, ${ }^{1}$ A. V. Timoshkin, ${ }^{2,3}$ Tanmoy Paul $^{4,5}$ \\ 1) Department of Energy and Process Engineering, \\ Norwegian University of Science and Technology, N-7491 Trondheim, Norway \\ 2) Tomsk State Pedagogical University, Kievskaja Street, 60, 634061 Tomsk, Russia \\ 3) International Laboratory of Theoretical Cosmology, \\ Tomsk State University of Control Systems and Radio Electronics, Lenin Avenue, 36, 634050 Tomsk, Russia \\ 4) Department of Physics, Chandernagore College, Hooghly - 712136. \\ (5) Department of Theoretical Physics, Indian Association for the Cultivation of Science, \\ $2 A$ \& $2 B$ Raja S.C. Mullick Road, Kolkata - 700 032, India
}

\begin{abstract}
Cosmological models with an inhomogeneous viscous dark fluid, coupled with dark matter in the Friedmann- Robertson-Walker (FRW) flat universe, are considered. The influence of thermal effects caused by Hawking radiation on the visible horizon is studied, in connection with the classified type I and type III singularities which are known to occur within a finite amount of time. Allowance of thermal effects implies that a transition to a type II singularity can take place, in a finite time. We take into account a bulk viscosity of the dark fluid, observing the equation of state in the case of radiation, and find that there is a qualitative change in the singular universe of type I: it may pass into a singularity of type III, or it may avoid the singularity at all.
\end{abstract}

\section{INTRODUCTION}

After it was observed that the universe is exposed to an accelerated expansion, it is of great interest to study the nature of dark energy which is responsible for this acceleration [1-4]. In the era of dark energy the universe can be qualitatively described using an exotic effective fluid with negative pressure (more appropriately called a positive tensile stress), that satisfies an unusual equation of state [5-13]. Dark energy can be characterized by an equation of state parameter $\omega_{\text {eff }}=p_{\text {eff }} / \rho_{\text {eff }}$. Depending on the values of $\omega_{\text {eff }}$ several possibilities of the universe are possible: for example, phantom behaviour occurs for $\omega_{\text {eff }}<-1$, de-Sitter evolution occurs for $\omega_{\text {eff }}=-1$, and quintessential behaviour occurs for $-1<\omega_{\text {eff }}<-1 / 3$. The experimental value of the parameter of the equation of state is however determined with insufficient accuracy to be able to unambiguously determine the phase in which our Universe is located. Today the value of this parameter lies within the following limits $\omega_{\text {eff }}=1.04_{-0.10}^{+0.09}$ [14].

One of the properties of phantom dark energy is the prediction of a Big Rip singularity (type I) in the future. For this kind of singularity the scale factor and the Hubble function go to infinity at a finite time, called $t_{s}$ or $t_{\text {rip }}$. This is the most destructive type of singularity [15]. There are several less drastic future singularities, namely soft singularities classified as type II, III and IV. All these singularities are purely classic in nature.

From a physical viewpoint, an increase in the Hubble function should be expected to lead to an increase in temperature. At high temperatures, especially near the singularity, thermal radiation should appear. Thermal radiation is associated with Hawking radiation, which effectively should be generated at the apparent horizon of the FRW universe [16]. Hawking's radiation is manifested in black holes and is associated with the existence of the visible black hole horizon, as well as the visible horizon of cosmic events in de-Sitter space. Hawking's thermal spectrum radiation should be taken to appear in the late universe at high temperatures shortly before its rupture. Accounting for thermal radiation will allow a qualitative change in the classical description and give a more realistic picture of the future of the universe.

Recently, the effect of thermal radiation on future singularities of types I, II, III and IV was studied in Ref. [17]. It was shown that with singular universes of types I and III, as well as for the Little Rip universe, there occurs a qualitative change in the singularity due to thermal effects. The singularities end up as type II singularities. In universes of types II and IV there is no qualitative change in the final state.

The model of a non-viscous fluid in cosmology is an idealized case. Dark energy universe with a viscous fluid was studied in Refs. [18 26]. The cosmic viscosity property was taken into account in connection with the Big Rip type singularity [27], and also in connection with the singularities of type II, III and IV [28, 29]. Models of dark energy interacting with dark matter, in which the singularities of one of these four types are formed, were considered in Ref. 30, 31].

The article [32] studied a combined phantom/fluid model consisting of a viscous dark fluid (dark energy) with a linear inhomogeneous equation of state and dark matter with a linear homogeneous equation of state in a spatially flat FRW metric.

The purpose of the present article is to study the influence of thermal radiation, taking into account the interaction of viscous dark fluid with dark matter. In particular, we will focus on the change of singular behavior of the late-time 
universe.

\section{THE EFFECT OF THERMAL RADIATION ON THE FORMATION OF SINGULARITIES IN THE LATE UNIVERSE}

Let us consider a spatially-flat FRW universe

$$
d s^{2}=-d t^{2}+a^{2}(t) \delta_{i j} d x^{i} d x^{j}
$$

where $a(t)$ is the scale factor. We will be interested in the case when the effective parameter in the equation of state takes values in the vicinity of -1 . Then the following kinds of evolution of the accelerating universe are possible : phantom, quintessence, or a de Sitter expansion. The question arises about how this evolution will end up in the future. The answer depends on the behavior of the time-dependent parameters of the effective equation of state. We will be interested in dark energy universes, in which there occur future singularities within a finite, or an infinite, time. For such universes the Nojiri-Odintsov-Tsujikawa classification was given in Ref. [5] (see also [33]). Singularities arise when one or more of the central cosmological parameters diverge: the scale factor $a(t)$, the effective (total) energy density $\rho_{\text {eff }}$, the effective (total) pressure $p_{\text {eff }}$, or higher derivatives of the Hubble function.

In the limit $t \rightarrow t_{s}$, the following classes of singularities can be distinguished:

- Type I (Big Rip): $a \rightarrow \infty, \rho_{\text {eff }} \rightarrow \infty$ and $p_{\text {eff }} \rightarrow \infty$. This class of singularities includes the case when $\rho_{\text {eff }}$ and $p_{\text {eff }}$ are finite at $t=t_{s}$. A Big Rip leads to the decay of gravitationally bound objects large on a cosmological scale.

- Type II ("sudden" singularity): $a \rightarrow a_{s}, \rho_{\text {eff }} \rightarrow \rho_{s}$ and $\left|p_{\text {eff }}\right| \rightarrow \infty$, where $a_{s} \neq 0$ and $\rho_{s}$ are constant. That is a pressure singularity.

- Type III: $a \rightarrow a_{s}, \rho_{\text {eff }} \rightarrow \infty$ and $p_{\text {eff }} \rightarrow \infty$. This type singularity is milder than Type I but stronger than Type II.

- Type IV $: a \rightarrow a_{s}, \rho_{\text {eff }} \rightarrow 0$ and $p_{\text {eff }} \rightarrow 0$, but the higher derivatives of the Hubble function $H$ diverge. This type also includes the case where $\rho_{\text {eff }}$ and/or $p_{\text {eff }}$ are finite for $t=t_{s}$.

Here $\rho_{\text {eff }}$ and $p_{\text {eff }}$ can be calculated by the following expressions,

$$
\rho_{\mathrm{eff}}=\frac{3}{\kappa^{2}} H^{2} \quad, \quad p_{\mathrm{eff}}=-\frac{2}{\kappa^{2}}\left(2 \dot{H}+3 H^{2}\right)
$$

where $\kappa^{2}=8 \pi G$ and $H=\frac{\dot{a}}{a}$ is the Hubble parameter. It may be mentioned that the effective energy density $\rho_{\text {eff }}$ and the effective pressure $p_{\text {eff }}$ may include the contribution from the modified gravity.

However, the singularity is not the only possible outcome of the evolution of our universe in the phantom phase. It was shown in [33 36] that if the cosmic energy density remains constant or monotonically increases, then, depending on the asymptotic behavior of the Hubble parameter $H$ [33], all possible types of evolution of our universe can be divided into four categories:

- Big Rip: $H(t) \rightarrow \infty$ when $t=t_{s}<\infty$.

- Little Rip: $H(t) \rightarrow \infty$ when $t \rightarrow \infty$.

- Cosmological constant : $H(t)=$ constant.

- Pseudo Rip: $H(t) \rightarrow H_{\infty}$ when $t \rightarrow \infty$, where $H_{\infty}$ is a constant.

Here we would like to mention that both the Little Rip and Pseudo Rip models are nonsingular.

We will study the cosmological models induced by the inhomogeneous viscous dark fluids coupled with dark matter, in terms of the parameters appearing in the equation of state (EoS). Let us consider the following formulation of the EoS of an inhomogeneous viscous fluid in flat FRW space-time [37], namely

$$
p=\omega(\rho, t) \rho-3 H \xi(H, t)
$$

where $\xi(H, t)$ is the bulk viscosity, which depends on the Hubble parameter $H$ and on the cosmic time $t$. According to the thermodynamic set up, we naturally assume that $\xi(H, t)>0$. 
We will take the following form for the thermodynamic (EoS) parameter $\omega$ [37],

$$
\omega(\rho, t)=\omega_{1}(t)\left(A_{0} \rho^{\alpha-1}-1\right)
$$

where $A_{0} \neq 0$ and $\alpha \geq 1$ are constants. (Note that $A_{0}$ is nondimensional only if $\alpha=1$. If $\alpha=3 / 2$ for instance, the dimension will be $\mathrm{cm}^{2}$ in geometric units.)

We choose the bulk viscosity as [37],

$$
\xi(H, t)=\xi_{1}(t)(3 H)^{n}
$$

with $n>0$.

Let us consider the influence of thermal effects on the change in singularities of types I, taking into account the viscosity property of a dark fluid and its interaction with dark matter. Since the temperature of the universe increases near the singularity, thermal radiation is generated, as mentioned above. From statistical physics, the energy density of thermal radiation is proportional to the fourth power of the absolute temperature. Therefore, near the future singularity where the Hubble parameter becomes very high, we assume that the thermal energy density has the form [17]

$$
\rho_{\mathrm{rad}}=\lambda H^{4}
$$

where $\lambda$ is a positive constant.

Taking thermal radiation into account, the FRW equation is modified as follows [17],

$$
\frac{3}{\kappa^{2}} H^{2}=\rho_{\text {eff }}+\lambda H^{4}
$$

From Eq.(17) it follows that when the evolution time of the late universe is much less than the singularity time, the first term of the equation makes the greatest contribution. While near the singularity time, the second term makes the largest contribution. We analyze equation (7) further, by solving it with respect to the square of the Hubble parameter $H^{2}$

$$
H^{2}=\frac{1}{2 \lambda}\left[\frac{3}{\kappa^{2}} \pm \sqrt{\frac{9}{\kappa^{4}}-4 \lambda \rho_{\mathrm{eff}}}\right] .
$$

In the following, we will apply the cosmological models of a viscous fluid from the article [32].

\section{SINGULAR BEHAVIOR OF LATE-TIME UNIVERSE TAKING INTO ACCOUNT THE VISCOSITY OF A FLUID AND ITS INTERACTION WITH DARK MATTER}

We start from the simplest, constant case, namely $\omega(\rho, t)=\omega_{0}$ and will consider different forms for the bulk viscosity.

\section{A. Constant viscosity}

Let us consider the case of constant bulk viscosity $\xi(H, t)=\xi_{0}>0$. The Hubble function has the form 32 ]

$$
H(t)=\frac{\xi_{0} \kappa^{2}}{\left(1+\omega_{0}\right)\left(1-\sqrt{C_{1}} \exp \left[3 \xi_{0} \kappa^{2} t / 2\right]\right)},
$$

with $C_{1}$ a nondimensional constant. This model does not so far take into account interactions with dark matter. Observe that $H$ diverges for $t \rightarrow t_{s}=-\frac{2}{3 \xi_{0} \kappa^{2}} \ln \left(\sqrt{C_{1}}\right)$, thus a Big Rip singularity appears. Let us now see if the type of singularity will change if we take into account thermal radiation near the singularity time.

We calculate the scale factor,

$$
a(t)=e^{\int H d t}=a_{0}\left(1-\frac{1}{\sqrt{C_{1}}} \exp \left[-3 \xi_{0} \kappa^{2} t / 2\right]\right)^{\frac{2}{3\left(1+\omega_{0}\right)}}
$$


where $a_{0}$ is an integration constant. Then the effective energy density in terms of the scale factor is

$$
\rho_{\text {eff }}=\left(\sqrt{\frac{3}{\rho_{0}}} \frac{\xi_{0} \kappa}{1+\omega_{0}}\right)^{2}\left[\frac{a(t)}{a_{0}}\right]^{3\left(1+\omega_{0}\right)} e^{-3 \xi_{0} \kappa^{2} t} .
$$

Let us return to Eq.(8). Since $H^{2}$ is a real number, we must have

$$
\frac{9}{\kappa^{4}}-4 \lambda \rho_{\mathrm{eff}} \geq 0
$$

We get in this model

$$
\frac{9}{\kappa^{4}}-4 \lambda A e^{-B t}\left[\frac{a(t)}{a_{0}}\right]^{C} \geq 0
$$

where $A=\left(\sqrt{\frac{3}{\rho_{0}}} \frac{\xi_{0} \kappa}{1+\omega_{0}}\right)^{2}, B=3 \xi_{0} \kappa^{2}$ and $C=3\left(1+\omega_{0}\right)$. The above inequality puts a restriction on the scale factor,

$$
a(t) \leq a_{0}\left(\frac{9}{4 \lambda A \kappa^{4}}\right)^{\frac{1}{C}} e^{\frac{B}{C} t}
$$

under the condition $C<0\left(\omega_{0}<-1\right)$, which corresponds to the case of phantom dark energy.

Taking into account the thermal radiation $\rho_{\text {rad }}$, we obtain from Eq. (8) that there exists another upper limit $a_{\text {max }}$ for the scale parameter,

$$
a(t) \leq a_{\max }=a_{0}\left(\frac{4 \lambda A \kappa^{4}}{9}\right)^{\frac{1}{3\left(1+\omega_{0}\right)}},
$$

which corresponds to the instant $t_{\max }$,

$$
t_{\max }=-\frac{2}{3 \xi_{0} \kappa^{2}} \ln \left\{\sqrt{C_{1}}\left(1-\frac{2 \kappa^{2} \sqrt{A \lambda}}{3}\right)\right\} .
$$

This is thus a singularity of another type than that arising from the Hawking radiation.

We calculate the difference between $t_{\max }$ and $t_{s}$

$$
t_{\max }-t_{s}=-\frac{2}{3 \xi_{0} \kappa^{2}} \ln \left\{1-\frac{2 \kappa^{2} \sqrt{A \lambda}}{3}\right\}>0,
$$

which shows that $t_{\max }$ is larger than $t_{s}$. In the limit $t \rightarrow t_{\max }, a \rightarrow a_{\max }$. From Eqs.(2) and (3), one can calculate the effective energy density $\rho_{\text {eff }}$ and the effective pressure $p_{\text {eff }}$. Then in the limit $t \rightarrow t_{\max }$, the effective energy density and effective pressure become

$$
\rho_{\max }=\rho_{\mathrm{eff}}\left(t_{\max }\right)=\frac{9 \xi_{0}^{2} \kappa^{2}}{\left(1+\omega_{0}\right)^{2}}\left[\frac{1}{1+C_{1}\left(\frac{2 \kappa^{2} \sqrt{A \lambda}}{3}-1\right)}\right]^{2},
$$

and

$$
p_{\max }=p_{\text {eff }}\left(t_{\max }\right)=\left|\omega_{0} \rho_{\max }-3 \xi_{0} H_{\max }\right|=\frac{3 \xi_{0}^{2} \kappa^{2}}{\left(1+\omega_{0}\right)}\left\{\frac{\left|\frac{2 \omega_{0}-1}{1+\omega_{0}}-C_{1}\left(\frac{2 \kappa^{2} \sqrt{A \lambda}}{3}-1\right)\right|}{\left[1+C_{1}\left(\frac{2 \kappa^{2} \sqrt{A \lambda}}{3}-1\right)\right]^{2}}\right\} .
$$

respectively. In the general case the values of the scale factor, energy density and effective pressure turn out to be finite, but higher derivatives of $\mathrm{H}$ diverge. Thus, a cosmological finite-time future singularity is not formed. This behavior is due to the influence from the viscosity of the dark fluid, which compensates for the effect of thermal radiation. However, if the radiation parameter $\lambda$ goes to $\lambda_{0}$, with

$$
\lambda_{0}=\frac{3}{C_{1}}\left(\frac{\left(C_{1}-1\right)\left(1+\omega_{0}\right)}{2 \xi_{0} \kappa^{3}}\right)^{2},
$$

then $\rho_{\max } \rightarrow \infty$ and $\left|p_{\max }\right| \rightarrow \infty$. This is a type III singularity. It is milder than type I, but stronger than type II. If $\zeta_{0} \rightarrow \infty$, then $\lambda_{0} \rightarrow 0$. Thus, the viscosity weakens the effect of thermal radiation. 


\section{B. Viscosity proportional to the Hubble parameter}

Let us consider the case where the viscosity is proportional to the Hubble parameter, $\xi(H, t)=3 \tau H$, the constant $\tau$ being positive. The Hubble parameter becomes [32]

$$
H(t)=\frac{\kappa}{\sqrt{3}}\left\{\frac{\delta \gamma \sqrt{C_{1}}}{3 \theta \sqrt{C_{1}}+\exp [-\tilde{\eta} t / 2]}\right\} .
$$

Here the following designations are introduced:

$$
\eta=\delta \gamma^{2}, \quad \gamma=\frac{\kappa}{\sqrt{3}} \sqrt{1+\frac{1}{r}}, \quad \frac{\tilde{\eta}}{\eta}=r, \quad \theta=1+\omega_{0}-9 \tau \gamma^{2},
$$

where the constant $r$ is associated with the influence of dark matter and is equal to the ratio of the energy density of dark matter to the density of dark energy. The dimensions are $[\gamma]=\mathrm{cm},[\eta]=[\tilde{\eta}]=\mathrm{cm}^{-1},[\delta]=\mathrm{cm}^{-2},[\tau]=\mathrm{cm}^{-2}$. Further, the constant parameter $\delta$ is responsible for the interaction with dark matter. If $\omega_{0}<-1+9 \tau \gamma^{2}$, then $\theta<0$ and consequently $H$ diverges at $t \rightarrow t_{s}=-\frac{2}{\tilde{\eta}} \ln \left(-3 \theta \sqrt{C_{1}}\right)$, which leads to the appearance of a singularity of the type Big Rip. We consider again the behavior of the late-time universe near the singularity, taking into account the effect of thermal radiation.

Let us calculate the scale factor,

$$
a(t)=a_{0} \exp \left[\frac{\delta \gamma \kappa t}{3 \sqrt{3} \theta}\right]\left(3 \theta \sqrt{C_{1}}+\exp [-\tilde{\eta} t / 2]\right)^{\frac{2 \delta \gamma \kappa}{3 \sqrt{3} \theta \tilde{\eta}}}
$$

and express the effective energy density in terms of the scale factor as

$$
\rho_{\text {eff }}=C_{1}(\delta \gamma)^{2}\left[\frac{a(t)}{a_{0}}\right]^{-\frac{2}{\delta}} \exp \left[\frac{2 \alpha^{\prime}}{\tilde{\delta}} t\right]
$$

where $\alpha^{\prime}=\frac{\delta \gamma \kappa}{3 \sqrt{3} \theta}$ and $\tilde{\delta}=\frac{2 \delta \gamma \kappa}{3 \sqrt{3} \theta}$. The inequality in Eq.(12) implies the following restriction on the scale factor,

$$
a(t) \leq a_{0}\left(\frac{2 \delta \gamma \kappa^{2} \sqrt{\lambda C_{1}}}{3}\right)^{\tilde{\delta}} e^{\alpha^{\prime} t}
$$

Since $\theta<0$, then $\alpha^{\prime}<0$ and scale factor values are limited by the maximum number $a_{\max }$ given by

$$
a(t) \leq a_{\max }=a_{0}\left(\frac{2 \delta \gamma \kappa^{2} \sqrt{\lambda C_{1}}}{3}\right)^{\tilde{\delta}}
$$

which corresponds to the instant

$$
t_{\max }=\frac{2}{\tilde{\eta}} \ln \left\{\frac{2 \delta \gamma \kappa^{2} \sqrt{\lambda C_{1}}-3}{9 \theta \sqrt{C_{1}}}\right\}
$$

Let us find the difference between $t_{s}$ and $t_{\max }$,

$$
t_{s}-t_{\max }=-\frac{2}{\tilde{\eta}} \ln \left\{1-\frac{2}{3} \delta \gamma \kappa^{2} \sqrt{\lambda C_{1}}\right\}>0
$$

Hence it follows that $t_{s}$ is larger than $t_{\max }$. Thus in the limit $t \rightarrow t_{\max }$, the effective energy density and the effective pressure are given by

$$
\rho_{\max }=\rho_{\mathrm{eff}}\left(t_{\max }\right)=\frac{1}{\theta^{2}}\left(\delta \gamma-\frac{1}{\kappa^{2} \sqrt{\lambda C_{1}}}\right)^{2}
$$

and

$$
\left|p_{\max }\right|=\left|p\left(t_{\max }\right)\right|=\left|\omega_{0} \rho_{\max }-9 \tau H_{\max }^{2}\right|=\left|\omega_{0}-3 \tau \kappa^{2}\right| \rho_{\max }
$$


respectively. In the limit when the radiation parameter $\lambda \rightarrow \lambda_{0}$ where $\lambda_{0}$ is given by

$$
\lambda_{0}=\left(\frac{3}{\sqrt{\rho_{0}} \delta \tilde{\gamma} \kappa^{2}}\right)^{2}
$$

$\rho_{\max } \rightarrow 0$ and $\left|p_{\max }\right| \rightarrow 0$. However, the higher derivatives of the Hubble function do not diverge. Thus, the formation of a singularity of IV type does not occur in this model.

We see that viscosity softens the singularity or avoids it altogether. This is due to a decrease in the pressure of a viscous fluid due to the viscosity term in the equation of state.

\section{Inhomogeneous fluid with variable parameter $\omega$}

In this section we will assume that the thermodynamic parameter $\omega(\rho, t)$ is a function of the energy density of the fluid. Let us choose it to have the form

$$
\omega(\rho, t)=A_{0} \rho^{\alpha-1}-1
$$

where $A_{0} \neq 0$ is a dimensional constant. The bulk viscosity is taken to be proportional to $H^{n}$,

$$
\xi(H, t)=\tau(3 H)^{n}
$$

with $\tau$ and $n$ positive. In the case $n=2 \alpha-1$, the energy density becomes [32]

$$
H(t)=H_{0}\left\{\rho_{0} \exp \left[\left(\alpha-\frac{1}{2}\right) \eta t\right]+\frac{\mu}{\tilde{\eta}}\right\}^{\frac{2}{1-2 \alpha}} \quad, \quad H_{0}=\frac{\kappa}{\sqrt{3}} \sqrt{\rho_{0}}
$$

where $\mu$ is a dimensionless constant and $\alpha \neq \frac{1}{2}$. Here $t_{0}$ is the present time, and $\rho_{0}=\rho\left(t_{0}\right)$, The dimension is $\left[\rho_{0}\right]=\mathrm{cm}^{-4}$.

For $\alpha>\frac{1}{2}$, then at $t \rightarrow t_{s}=\frac{1}{\tilde{\eta}(\alpha-1 / 2)} \ln \left(-\frac{\mu}{C_{1} \tilde{\eta}}\right)$, the Hubble parameter diverges and we obtain again the Big Rip singularity. Further, if we consider the case $\alpha=3 / 2$ the scale factor turns out to be

$$
a(t)=a_{0}\left(1+\frac{\mu}{C_{1} \tilde{\eta}} e^{-\eta t}\right)^{\frac{r H_{0}}{\mu}}
$$

and consequently the energy density in terms of the scale factor becomes

$$
\rho_{\mathrm{eff}}=\rho_{0}\left(C_{1} e^{\eta t}\right)^{-2}\left[\frac{a(t)}{a_{0}}\right]^{\frac{2 \mu}{r H_{0}}}
$$

The inequality in Eq.(12) implies the following restriction on the scale factor,

$$
a(t) \leq a_{0}\left(\frac{2 \kappa^{2} \sqrt{\lambda \rho_{0}}}{3 C_{1}}\right)^{\frac{r H_{0}}{\mu}} \exp \left[\frac{r H_{0} \eta t}{\mu}\right]
$$

Scale factor values are limited by the maximum number $a_{\max }$,

$$
a(t) \leq a_{\max }=a_{0}\left(\frac{2 \kappa^{2} \sqrt{\lambda \rho_{0}}}{3 C_{1}}\right)^{\frac{r H_{0}}{\mu}}
$$

which corresponds to the time

$$
t_{\max }=\frac{1}{\eta} \ln \frac{3 \mu}{\tilde{\eta}\left(2 \kappa^{2} \sqrt{\lambda \rho_{0}}-3 C_{1}\right)} .
$$

The difference between $t_{s}$ and $t_{\text {max }}$ takes the following form,

$$
t_{s}-t_{\max }=\frac{1}{\eta} \ln \left(1-\frac{2 \kappa^{2}}{3 C_{1}} \sqrt{\lambda \rho_{0}}\right)<0 .
$$


It turns out that $t_{\max }$ is larger than $t_{s}$. Thus, it due to thermal radiation, the time of formation of the cosmological singularity changes qualitatively. In the limit $t \rightarrow t_{\max }$, the effective energy density and the effective pressure are given by

$$
\rho_{\max }=\rho_{\mathrm{eff}}\left(t_{\max }\right)=\rho_{0}\left[\frac{\tilde{\eta}}{\mu}\left(1-\frac{3 C_{1}}{2 \kappa^{2} \sqrt{\lambda \rho_{0}}}\right)\right]
$$

and

$$
\left|p_{\max }\right|=\left|p\left(t_{\max }\right)\right|=\left|\left(A_{0} \rho_{\max }^{\frac{1}{2}}-1\right) \rho_{\max }-\tau\left(3 H_{\max }\right)^{3}\right|=\left|\left(A_{0}-3 \sqrt{3} \tau \kappa^{3}\right) \rho_{\max }^{\frac{1}{2}}-1\right| \rho_{\max },
$$

respectively. Thereby, the value of the energy density and effective pressure becomes finite, while higher derivatives of the Hubble function diverge. Consequently, a cosmological singularity is not formed.

\section{CONCLUSION}

We have investigated the singular behavior of the dark universe, taking into account the thermal effects caused by Hawking radiation, the viscosity properties of the dark fluid, and its interaction with dark matter on the visible horizon of the FRW universe. According to the study carried out in [17] for an ideal fluid, near the singularity it is necessary to take into account the Hawking thermal radiation, which leads to a change in the type of the singularity. In a dark universe with singularities of types I and III with a finite formation time, a transition to a type II singularity occurs.

Models with an inhomogeneous viscous dark fluid interacting with dark matter were considered in 32 . It is shown that, in our case, a transition from singularity of type I to singularity III type is possible due to the influence of thermal radiation. Singularities may be absent, due to the viscosity of the dark fluid and its interaction with dark matter. The absence of a singularity in some models is explained by the fact that both the presence of a bulk viscosity in the equation of state of a dark fluid, and the presence of a thermal radiation term in the Friedmann equation, are proportional to a power of the Hubble parameter. As a result, the effect of thermal radiation near the singularity may be neutralized by a viscous fluid.

As was shown in Ref. [38], taking thermal radiation into account does not weaken the agreement of cosmological models with astronomical obeservations.

\section{Acknowledgments}

This work was supported in part by Ministry of Education of Russian Federation, Project No FEWF-2020-0003 (A.V.T.).

[1] S. Nojiri, S. D. Odintsov and V. K. Oikonomou, Modified gravity theories on a nutshell: Inflation, bounce and late-time evolution, Phys. Rept. 692 (2017), 1-104 doi:10.1016/j.physrep.2017.06.001 arXiv:1705.11098 [gr-qc]].

[2] Y. Shi, A cyclic cosmological model based on the $f(\rho)$ modified theory of gravity, arXiv:1106.0341 [physics.gen-ph]].

[3] L. Perivolaropoulos, Accelerating universe: observational status and theoretical implications, AIP Conf. Proc. 848 (2006) no.1, 698-712 doi:10.1063/1.2348048 arXiv:astro-ph/0601014 [astro-ph]].

[4] I. Y. Aref'eva and I. V. Volovich, On the null energy condition and cosmology, Theor. Math. Phys. 155 (2008), 503-511 doi:10.1007/s11232-008-0041-8 arXiv:hep-th/0612098 [hep-th]].

[5] S. Nojiri, S. D. Odintsov and S. Tsujikawa, Properties of singularities in (phantom) dark energy universe, Phys. Rev. D 71 (2005), 063004 doi:10.1103/PhysRevD.71.063004 arXiv:hep-th/0501025 [hep-th]].

[6] S. Nojiri and S. D. Odintsov, The new form of the equation of state for dark energy fluid and accelerating universe, Phys. Lett. B 639 (2006), 144-150 doi:10.1016/j.physletb.2006.06.065 arXiv:hep-th/0606025 [hep-th]].

[7] S. Nojiri and S. D. Odintsov, The final state and thermodynamics of dark energy universe, Phys. Rev. D 70 (2004), 103522 doi:10.1103/PhysRevD.70.103522 arXiv:hep-th/0408170 [hep-th]].

[8] M. R. Setare, Interacting generalized Chaplygin gas model in non-flat universe, Eur. Phys. J. C 52 (2007), 689-692 doi:10.1140/epjc/s10052-007-0405-5 arXiv:0711.0524 [gr-qc]].

[9] S. Capozziello, S. Nojiri and S. D. Odintsov, Dark energy: The equation of state description versus scalar-tensor or modified gravity, Phys. Lett. B 634 (2006), 93-100 doi:10.1016/j.physletb.2006.01.065 arXiv:hep-th/0512118 [hep-th]]. 
[10] G. Cognola, E. Elizalde, S. Nojiri, S. D. Odintsov and S. Zerbini, Dark energy in modified Gauss-Bonnet gravity: Late-time acceleration and the hierarchy problem, Phys. Rev. D 73 (2006), 084007 doi:10.1103/PhysRevD.73.084007 arXiv:hep-th/0601008 [hep-th]].

[11] S. Nojiri and S. D. Odintsov, Inhomogeneous equation of state of the universe: Phantom era, future singularity and crossing the phantom barrier, Phys. Rev. D 72 (2005), 023003 doi:10.1103/PhysRevD.72.023003 arXiv:hep-th/0505215 [hep-th]].

[12] S. Nojiri, S. D. Odintsov, V. K. Oikonomou and T. Paul, Unifying holographic inflation with holographic dark energy: a covariant approach, Phys. Rev. D 102 (2020) no.2, 023540 doi:10.1103/PhysRevD.102.023540 arXiv:2007.06829 [gr-qc]].

[13] S. D. Odintsov, V. K. Oikonomou and T. Paul, From a bounce to the dark energy era with $F(R)$ gravity, Class. Quant. Grav. 37 (2020) no.23, 235005 doi:10.1088/1361-6382/abbc47 [arXiv:2009.09947] [gr-qc]].

[14] K. Nakamura et al. [Particle Data Group], Review of particle physics, J. Phys. G 37 (2010), 075021 doi:10.1088/0954$3899 / 37 / 7 \mathrm{~A} / 075021$

[15] R. R. Caldwell, A phantom menace?, Phys. Lett. B 545 (2002), 23-29 doi:10.1016/S0370-2693(02)02589-3 arXiv:astro-ph/9908168 [astro-ph]].

[16] R. G. Cai and N. Ohta, Horizon thermodynamics and gravitational field equations in Horava-Lifshitz Gravity, Phys. Rev. D 81 (2010), 084061 doi:10.1103/PhysRevD.81.084061 [arXiv:0910.2307 [hep-th]].

[17] S. Nojiri and S. D. Odintsov, The dark universe future and singularities: the account of thermal and quantum effects, Phys. Dark Univ. 30 (2020), 100695 doi:10.1016/j.dark.2020.100695 arXiv:2006.03946 [gr-qc]]; A. Astashenok, S. D. Odintsov and V. K. Oikonomou, submitted.

[18] Ø. Grøn, Viscous inflationary universe models, Astrophys. Space Sci. 173 (1990), 191-225 doi:10.1007/BF00643930

[19] I. Brevik, Ø. Grøn, J. de Haro, S. D. Odintsov and E. N. Saridakis, Viscous cosmology for early- and late-time Universe, Int. J. Mod. Phys. D 26 (2017) no.14, 1730024 doi:10.1142/S0218271817300245 arXiv:1706.02543 [gr-qc]].

[20] M. Cataldo, N. Cruz and S. Lepe, Viscous dark energy and phantom evolution, Phys. Lett. B 619 (2005), 5-10 doi:10.1016/j.physletb.2005.05.029 arXiv:hep-th/0506153 [hep-th]].

[21] I. Brevik, J. M. Børven and S. Ng, Viscous brane cosmology with a brane-bulk energy interchange term, Gen. Rel. Grav. 38 (2006), 907-915 doi:10.1007/s10714-006-0271-8 arXiv:gr-qc/0512026 [gr-qc]].

[22] I. Brevik and S. D. Odintsov, On the Cardy-Verlinde entropy formula in viscous cosmology, Phys. Rev. D 65 (2002), 067302 doi:10.1103/PhysRevD.65.067302 arXiv:gr-qc/0110105 [gr-qc]].

[23] I. Brevik, S. Nojiri, S. D. Odintsov and D. Saez-Gomez, Cardy-Verlinde formula in FRW Universe with inhomogeneous generalized fluid and dynamical entropy bounds near the future singularity, Eur. Phys. J. C 69 (2010), 563-574 doi:10.1140/epjc/s10052-010-1425-0 arXiv:1002.1942 [hep-th]].

[24] I. Brevik and O. Gorbunova, Dark energy and viscous cosmology, Gen. Rel. Grav. 37 (2005), 2039-2045 doi:10.1007/s10714005-0178-9 arXiv:gr-qc/0504001 [gr-qc]].

[25] B. Li and J. D. Barrow, Does bulk viscosity create a viable unified dark matter model?, Phys. Rev. D 79 (2009), 103521 doi:10.1103/PhysRevD.79.103521 arXiv:0902.3163 [gr-qc]].

[26] I. Brevik, E. Elizalde, S. D. Odintsov and A. V. Timoshkin, Inflationary universe in terms of a van der Waals viscous fluid, Int. J. Geom. Meth. Mod. Phys. 14 (2017) no.12, 1750185 doi:10.1142/S0219887817501857 [arXiv:1708.06244 [gr-qc]].

[27] S. Nojiri and S. D. Odintsov, Quantum de Sitter cosmology and phantom matter, Phys. Lett. B 562 (2003), $147-152$ doi:10.1016/S0370-2693(03)00594-X arXiv:hep-th/0303117 [hep-th]].

[28] J. D. Barrow and S. Cotsakis, Geodesics at sudden singularities, Phys. Rev. D 88 (2013), 067301 doi:10.1103/PhysRevD.88.067301 [arXiv:1307.5005 [gr-qc]].

[29] S. Nojiri and S. D. Odintsov, Quantum escape of sudden future singularity, Phys. Lett. B 595 (2004), 1-8 doi:10.1016/j.physletb.2004.06.060 arXiv:hep-th/0405078 [hep-th]].

[30] S. Myrzakul, R. Myrzakulov and L. Sebastiani, Inhomogeneous viscous fluids in FRW universe and finite-future time singularities, Astrophys. Space Sci. 350 (2014), 845-853 doi:10.1007/s10509-014-1799-9 arXiv:1311.6939 [gr-qc]].

[31] S. Capozziello, M. De Laurentis, S. Nojiri and S. D. Odintsov, Classifying and avoiding singularities in the alternative gravity dark energy models, Phys. Rev. D 79 (2009), 124007 doi:10.1103/PhysRevD.79.124007 arXiv:0903.2753 [hep-th]].

[32] E. Elizalde, V. V. Obukhov and A. V. Timoshkin, Inhomogeneous viscous dark fluid coupled with dark matter in the FRW universe, Mod. Phys. Lett. A 29 (2014) no.25, 1450132 doi:10.1142/S0217732314501326 arXiv:1406.7653 [gr-qc]].

[33] S. D. Odintsov and V. K. Oikonomou, Dynamical Systems Perspective of Cosmological Finite-time Singularities in $f(R)$ Gravity and Interacting Multifluid Cosmology, Phys. Rev. D 98 (2018) no.2, 024013 doi:10.1103/PhysRevD.98.024013 arXiv:1806.07295 [gr-qc]].

[34] P. H. Frampton, K. J. Ludwick and R. J. Scherrer, Pseudo-rip: Cosmological models intermediate between the cosmological constant and the little rip, Phys. Rev. D 85 (2012), 083001 doi:10.1103/PhysRevD.85.083001 arXiv:1112.2964 [astroph.CO]].

[35] P. H. Frampton, K. J. Ludwick and R. J. Scherrer, The Little Rip, Phys. Rev. D 84 (2011), 063003 doi:10.1103/PhysRevD.84.063003 arXiv:1106.4996 [astro-ph.CO]].

[36] P. H. Frampton, K. J. Ludwick, S. Nojiri, S. D. Odintsov and R. J. Scherrer, Models for Little Rip dark energy, Phys. Lett. B 708 (2012), 204-211 doi:10.1016/j.physletb.2012.01.048 arXiv:1108.0067 [hep-th]].

[37] S. Capozziello, V. F. Cardone, E. Elizalde, S. Nojiri and S. D. Odintsov, Observational constraints on dark energy with generalized equations of state, Phys. Rev. D 73 (2006), 043512 doi:10.1103/PhysRevD.73.043512 arXiv:astro-ph/0508350 [astro-ph]].

[38] V. Astashenok, S. D. Odintsov and V. K. Oikonomou, Dark energy and cosmological horizon thermal effects, Phys. Rev. D 103 (2021), 043514 doi:10.1103/PhysRevD.103.043514. 